\title{
A COMPARISON OF THE NEUROPROTECTIVE EFFICACY OF NEWLY DEVELOPED OXIMES (K156, K203) AND CURRENTLY AVAILABLE OXIMES (OBIDOXIME, HI-6) IN CYCLOSARIN-POISONED RATS
}

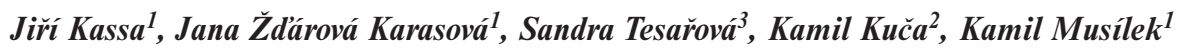

University of Defence, Faculty of Military Health Sciences, Hradec Králové, Czech Republic: Department of Toxicology ${ }^{1}$, Center of Advanced Studies ${ }^{2}$; The $7^{\text {th }}$ Field Hospital of the Czech Army, Hradec Králové, Czech Republic ${ }^{3}$

Summary: The neuroprotective effects of newly developed oximes (K156, K203) and currently available oximes (obidoxime, HI-6) in combination with atropine in rats poisoned with cyclosarin were studied. The cyclosarin-induced neurotoxicity was monitored using a functional observational battery 24 hours after cyclosarin challenge. The results indicate that a newly developed oxime K156 is able to counteract slightly cyclosarin-induced neurotoxicity while another newly developed oxime K203 is completely ineffective in reducing cyclosarin-induced neurotoxic signs and symptoms. The neuroprotective efficacy of K156 is comparable with commonly used obidoxime and oxime HI-6. Thus, none of the newly developed oximes achieves better neuroprotective efficacy than both commonly used oximes. They are therefore not suitable replacements for antidotal treatment of acute poisonings with cyclosarin.

Key words: Cyclosarin; K156; K203; Obidoxime; HI-6; Funtional observational battery; Rats

\section{Introduction}

Highly toxic organophosphorus compounds called nerve agents are considered to be the most dangerous chemical warfare agents. Their acute toxic effects is based on phosphylation of acetylcholinesterase (AChE, EC 3.1.1.7) leading to the irreversible inhibition of this enzyme and subsequent overstimulation of postsynaptic cholinergic receptors due to the accumulation of the neurotransmitter acetylcholine in synapses of the central and peripheral nervous systems $(1,13,15)$. The current medical countermeasures of nerve agent poisonings include the administration of special medicaments called antidotes which are able to counteract the main toxic effects of nerve agents. The current standard antidotal treatment of poisoning with nerve agents usually includes a muscarinic cholinergic receptor antagonist (preferably atropine) to block the overstimulation of cholinergic receptors by acetylcholine and an oxime to reactivate nerve agent-inhibited $\operatorname{AChE}(5,22)$.

Nerve agents can produce centrally-mediated seizure activity which rapidly progresses to the status of epilepsy and contributes to profound brain damage $(15,22)$. Therefore, the ability of antidotes to block the acute neurotoxic effects of nerve agents and prevent the development of irreversible lesions in the central nervous system (CNS) is important for successful antidotal treatment. Generally, oximes exert more potent effects in the peripheral compared to the cen- tral system due to their poor penetration into the CNS. Nevertheless, there are published results demonstrating the penetration of oximes into the CNS and subsequent reactivation of nerve agent-inhibited $\mathrm{AChE}$ in the brain $(2,21)$. Although the rate of reactivation of nerve agent-inhibited $\mathrm{AChE}$ in the brain is lower compared to the peripheral system, the role of reactivation of nerve agent-inhibited AChE in the brain is important for survival from nerve agent exposure $(1,5)$.

Cyclosarin (cyclohexyl methylphosphonofluoridate) is among the traditional nerve agents. The new interest in the treatment of cyclosarin poisoning results from the identification of weaponised cyclosarin following the initial coalition invasion of Iraq during the first Gulf war in 1991. As the ability of currently used monopyridinium (e.g. pralidoxime) and bispyridinium oximes (e.g. obidoxime) to ameliorate cyclosarin-induced toxic signs (including centrally mediated seizure activity and tonic-clonic convulsions) is not fully sufficient (5), the replacement of commonly used oximes with a more effective oxime has been a long-standing goal for the treatment of nerve agent poisonings. Recently, the new bispyridinium oximes K156 [1-(4-hydroxyiminomethylpyridinium)-3-pyridiniumpropane dibromide] and K203 [1(4-carbamoylpyridinium)-4-(4-hydroxyiminomethylpyridini um)-but-2-ene dibromide] (Fig. 1) were primarily synthesized at our department $(11,19)$ to improve the efficacy of antidotal treatment in eliminating tabun-induced acute to- 
xicity. As the evaluation of the efficacy of both oximes (especially K203) against tabun brought promising results $(7,19)$, we decided to evaluate the efficacy of both K oximes against another nerve agent, cyclosarin, because we are still searching for a broad-spectrum oxime able to sufficiently counteract acute toxicity of all nerve agents.

The aim of this study was to compare the neuroprotective potency of two newly developed oximes (K156, K203) with currently available oximes (obidoxime, HI-6) in combination with an anticholinergic drug atropine in cyclosarin-poisoned rats. The cyclosarin-induced neurotoxic signs and symptoms were determined using a functional observational battery, a non-invasive and relatively sensitive type of neurological examination for a wide range of neurobiological functions including measurements of sensory, motor and autonomic nervous functions.

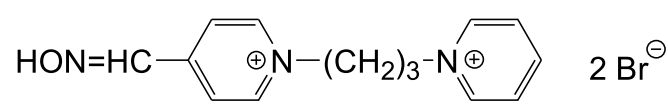

K156<smiles></smiles>

K203<smiles>O=[C+](CO)[n+]1ccc(C=NO)cc1</smiles>

Obidoxime<smiles>C[n+]1cccc(C(N)=O)c1</smiles>

HI-6

Fig. 1: Chemical structure of the oximes studied.

\section{Material and Methods}

Male albino Wistar rats weighing 180-200 g were purchased from Konarovice (Czech Republic). They were kept in an air-conditioned room $\left(22 \pm 2{ }^{\circ} \mathrm{C}\right.$ and $50 \pm 10 \%$ relative humidity, with light from 7.00 to 19.00 hours) and allowed access to standard food and tap water ad libitum. The rats were divided into groups of eight animals $(\mathrm{N}=8)$. Handling of the experimental animals was done under the supervision of the Ethics Committee of the Faculty of Military Health Sciences in Hradec Kralove (Czech Republic).

Cyclosarin was obtained from the Military Technical Institute in Brno (Czech Republic) and was $98.5 \%$ pure. Its purity was assayed by acidimetric titration. Obidoxime, the oxime HI-6 and the newly developed oximes (K156, K203) (Fig. 1) of $97.5 \%$ purity were synthesized earlier at the Department of Toxicology at the Faculty of Military Health Sciences in Hradec Kralove (Czech Republic). Their purity was analysed using HPLC. All other drugs and chemicals of analytical grade were obtained commercially and used without further purification. All substances were administered intramuscularly (i.m.) at a volume of $1 \mathrm{~mL} / \mathrm{kg}$ body weight (b.w.).

Cyclosarin was administered at a sublethal dose (120 $\mu \mathrm{g} / \mathrm{kg}$ b.w. $\left.-80 \% \mathrm{LD}_{50}\right)$. One minute following cyclosarin challenge, the rats were treated with atropine $(21 \mathrm{mg} / \mathrm{kg}$ b.w.) in combination with an oxime. The oximes were administered at equitoxic doses corresponding to $5 \%$ of their $\mathrm{LD}_{50}$ values: HI-6 (39 mg/kg b.w), obidoxime $(10.5 \mathrm{mg} / \mathrm{kg}$ b.w), K156 (6.4 mg/kg b.w) and K203 (16.3 mg/kg b.w). The neurotoxicity of cyclosarin was monitored using the functional observational battery 24 hours after cyclosarin poisoning. The evaluated markers of cyclosarin-induced neurotoxicity in the experimental animals were compared with the parameters obtained from control rats to which saline was administered instead of cyclosarin and antidotes at the same volume ( $1 \mathrm{~mL} / \mathrm{kg}$ b.w.).

The functional observational battery (FOB) consists of 47 measurements of sensory, motor and autonomic nervous functions. Some of them are scored (Tab. 1), the others are measured in absolute units $(3,4,18)$. The first evaluation was obtained when cyclosarin-poisoned rats were in the home cage. The observer evaluated each animal s posture, palpebral closure and involuntary motor movements. Then, each rat was removed from the home cage and briefly handheld. The exploratory activity, piloerection and other skin abnormalities were noted. Salivation and nose secretion were also registered and scored. Then, the rats were placed on a flat surface which served as an open field. A timer was started for 3 minutes during which the frequency of rearing responses was recorded. At the same time, gait characteristics were noted and ranked, and arousal, stereotypy and bizarre behaviors and abnormal posture were evaluated. At the end of the third minute, the number of fecal boluses and urine pools on the adsorbent pad was registered. Reflex testing comprising recording each rat's response to a fron- 
Tab. 1: Functional observational battery (FOB).

\begin{tabular}{|c|c|c|c|c|c|c|c|c|c|c|}
\hline Marker & & & & & Scored va & lues only & & & & \\
\hline & -2 & -1 & 0 & 1 & 2 & 3 & 4 & 5 & 6 & 7 \\
\hline POSTURE & & & & $\begin{array}{l}\text { sitting or } \\
\text { standing }\end{array}$ & rearing & asleep & flattened & $\begin{array}{l}\text { lying on } \\
\text { side }\end{array}$ & $\begin{array}{c}\text { crouched } \\
\text { over }\end{array}$ & $\begin{array}{c}\text { head } \\
\text { bobbing }\end{array}$ \\
\hline CATCH DIFFICULTY & & & & passive & normal & defense & flight & escape & $\begin{array}{l}\text { aggrres- } \\
\text { sion }\end{array}$ & \\
\hline EASE OF HANDLING & & & & very easy & easy & \begin{tabular}{|c|} 
modera- \\
telly \\
difficult \\
\end{tabular} & difficult & & & \\
\hline MUSCULAR TONUS & atonia & $\begin{array}{c}\text { hypoto- } \\
\text { nia }\end{array}$ & normal & $\begin{array}{l}\text { hyper- } \\
\text { tonia }\end{array}$ & rigidity & $\begin{array}{c}\text { fascicula- } \\
\text { tions }\end{array}$ & & & & \\
\hline LACRIMATION & & & none & slight & severe & crusta & $\begin{array}{c}\text { coloured } \\
\text { crusta }\end{array}$ & & & \\
\hline PALPEBRAL CLOSURE & & & & open & slightly & half-way & \begin{tabular}{|c|} 
comple- \\
tely shud
\end{tabular} & ptosis & & \\
\hline ENDO-EXOPHTHALMUS & & endo & normal & exo & & & & & & \\
\hline PILOERECTION & & & no & yes & & & & & & \\
\hline SKIN ABNORMALITIES & & & normal & pale & erythema & cyanosis & pigmented & cold & injury & \\
\hline SALIVATION & & & none & slight & severe & & & & & \\
\hline NOSE SECRETION & & & none & slight & severe & coloured & & & & \\
\hline TONIC MOVEMENTS & & & normal & \begin{tabular}{|c|} 
contrac- \\
tion of \\
extensors
\end{tabular} & \begin{tabular}{|c|} 
opis- \\
thotonus
\end{tabular} & $\begin{array}{c}\text { empros- } \\
\text { thotonus }\end{array}$ & \begin{tabular}{|c|} 
explosive \\
jumps
\end{tabular} & \begin{tabular}{|c|} 
tonic \\
convul- \\
sions \\
\end{tabular} & & \\
\hline GAIT & & & normal & ataxia & $\begin{array}{c}\text { overcom- } \\
\text { pensation } \\
\text { of hind- } \\
\text { limbs } \\
\text { move- } \\
\text { ments }\end{array}$ & $\begin{array}{c}\text { feet point } \\
\text { outwards } \\
\text { from } \\
\text { body }\end{array}$ & $\begin{array}{c}\text { forelimbs } \\
\text { are } \\
\text { extended }\end{array}$ & $\begin{array}{c}\text { walks on } \\
\text { tiptoes }\end{array}$ & $\begin{array}{c}\text { hunched } \\
\text { body }\end{array}$ & $\begin{array}{c}\text { body is } \\
\text { flattened } \\
\text { against } \\
\text { surface }\end{array}$ \\
\hline GAIT SCORE & & & & normal & \begin{tabular}{|c|} 
slightly \\
impaired
\end{tabular} & $\begin{array}{c}\text { somewhat } \\
\text { impaired }\end{array}$ & \begin{tabular}{|c|} 
totally \\
impaired
\end{tabular} & & & \\
\hline MOBILITY SCORE & & & & normal & \begin{tabular}{|c|} 
slightly \\
impaired
\end{tabular} & $\begin{array}{l}\text { somewhat } \\
\text { impaired }\end{array}$ & \begin{tabular}{|c|} 
totally \\
impaired
\end{tabular} & & & \\
\hline $\begin{array}{l}\text { AROUSAL (level of } \\
\text { unprovoked activity) }\end{array}$ & & & & very low & sporadic & reduced & normal & enhanced & $\begin{array}{c}\text { perma- } \\
\text { nent }\end{array}$ & \\
\hline TENSION & & & none & $\begin{array}{l}\text { partial } \\
\text { (ears) }\end{array}$ & stupor & & & & & \\
\hline STEREOTYPY & & & none & $\begin{array}{c}\text { head } \\
\text { weaving }\end{array}$ & $\begin{array}{c}\text { body } \\
\text { weaving }\end{array}$ & grooming & circling & others & & \\
\hline BIZZARE BEHAVIOR & & & none & head & body & $\begin{array}{c}\text { self-muti- } \\
\text { lation }\end{array}$ & $\begin{array}{c}\text { abnormal } \\
\text { move- } \\
\text { ments }\end{array}$ & others & & \\
\hline APPROACH RESPONSE & & & & $\begin{array}{c}\text { no } \\
\text { reaction }\end{array}$ & normal & \begin{tabular}{|c|} 
slow \\
reaction
\end{tabular} & \begin{tabular}{|c|} 
energetic \\
reaction
\end{tabular} & \begin{tabular}{|c|} 
exagge- \\
rated \\
reaction
\end{tabular} & & \\
\hline TOUCH RESPONSE & & & & $\begin{array}{c}\text { no } \\
\text { reaction }\end{array}$ & normal & $\begin{array}{c}\text { slow } \\
\text { reaction }\end{array}$ & $\begin{array}{l}\text { energetic } \\
\text { reaction }\end{array}$ & $\begin{array}{c}\text { exagge- } \\
\text { rated } \\
\text { reaction }\end{array}$ & & \\
\hline CLICK RESPONSE & & & & $\begin{array}{c}\text { no } \\
\text { reaction }\end{array}$ & normal & $\begin{array}{c}\text { slow } \\
\text { reaction }\end{array}$ & $\begin{array}{c}\text { energetic } \\
\text { reaction }\end{array}$ & $\begin{array}{c}\text { exagge- } \\
\text { rated } \\
\text { reaction }\end{array}$ & & \\
\hline TAIL-PINCH RESPONSE & & & & $\begin{array}{c}\text { no } \\
\text { reaction }\end{array}$ & normal & $\begin{array}{c}\text { slow } \\
\text { reaction }\end{array}$ & $\begin{array}{c}\text { energetic } \\
\text { reaction }\end{array}$ & \begin{tabular}{|c|} 
exagge- \\
rated \\
reaction \\
\end{tabular} & & \\
\hline PUPIL SIZE & & miosis & normal & mydriasis & & & & & & \\
\hline PUPIL RESPONSE & & & $\begin{array}{c}\text { no } \\
\text { reaction }\end{array}$ & $\begin{array}{c}\text { normal } \\
\text { reaction }\end{array}$ & & & & & & \\
\hline RIGHTING REFLEX & & & & normal & \begin{tabular}{|c|} 
slightly \\
uncoordin.
\end{tabular} & $\begin{array}{c}\text { lands on } \\
\text { side }\end{array}$ & \begin{tabular}{|c|}
$\begin{array}{c}\text { lands on } \\
\text { back }\end{array}$ \\
\end{tabular} & & & \\
\hline
\end{tabular}


tal approach of the blunt end of a pen, a touch of the pen to the posterior flank and to an auditory clic stimulus was also used. The response to a pinch on the tail and the ability of pupils to constrict in response to light were then assessed. These measures were followed by a test for the aerial righting reflex and by the measurements of forelimb and hindlimb grip strength, body weight, body temperature and finally hindlimb landing foot splay. The whole battery of tests required approximately 6-8 min per rat. The observer of behavior did not know about the experiment's design. Motor activity data were collected shortly after finishing the functional observational battery using an apparatus for testing spontaneous motor activity of laboratory animals (constructed at the Faculty of Military Health Sciences, Hradec Kralove, Czech Republic). The animals were placed for a short period (10 min) in the measuring cage and their movements (total, horizontal and vertical activity) were recorded.

Data collected with the FOB and motor activity assessment include categorial, ordinal and continuous values. Their statistical analyses were performed on a PC with a special interactive programme NTX (3). The categorial and ordinal values were formulated as contingency tables and judged consecutively by Chi-squared test of homoge- neity, Concordance-Discordance test and Kruskal-Wallis test, respectively. The continual data were assessed by successive statistical tests: CI for Delta, Barlett test for Equality of Variance, Williams test and Test for Distribution Functions. The results of the experimental groups were compared to the condition of the control rats. The differences were considered significant when $\mathrm{p}<0.05$.

\section{Results}

The results of the experiments related to the measurement of cyclosarin-induced neurotoxicity at 24 hours following cyclosarin poisoning are divided into three parts (activity and neuromuscular measures, sensorimotor and excitability measures and autonomic measures) (18) and summarized in Table 2a-c. Three non-treated cyclosarinpoisoned rats and cyclosarin-poisoned rats treated with atropine in combination with K203, two cyclosarin-poisoned rat treated with atropine in combination with K156 or obidoxime and one cyclosarin-poisoned rat treated with atropine in combination with HI-6 died within 24 hours of cyclosarin administration.

The evaluation of cyclosarin-induced neurotoxic signs at 24 hours following intoxication proved significant altera-

Tab. 2a: The values of cyclosarin-induced activity and neuromuscular neurotoxic markers measured at 24 hours following cyclosarin challenge according to the functional observational battery (No 1-2, 4-14 - scored values, No 3, 15-21 - values in absolute units). Statistical significance: * $\mathrm{p}<0.05$ (comparison with the control values). Applied abbreviations: A, atropine; RRF, air righting reflex; RRV, air righting reflex from vertical position; $x / M$, average or modus value; $\pm \mathrm{s}$, standard deviation; $n$, number of surviving animals.

\begin{tabular}{|c|c|c|c|c|c|c|c|c|c|c|c|c|c|}
\hline \multicolumn{2}{|r|}{24 hours } & \multicolumn{2}{|c|}{ Controls } & \multicolumn{2}{|c|}{$\begin{array}{c}\text { Cyclosarin + A } \\
\text { + K } 156\end{array}$} & \multicolumn{2}{|c|}{$\begin{array}{c}\text { Cyclosarin + A } \\
\text { + K } 203\end{array}$} & \multicolumn{2}{|c|}{$\begin{array}{c}\text { Cyclosarin + A } \\
\text { + Obidoxime }\end{array}$} & \multicolumn{2}{|c|}{$\begin{array}{c}\text { Cyclosarin + A } \\
+ \text { HI-6 }\end{array}$} & \multicolumn{2}{|c|}{ Cyclosarin } \\
\hline No & Marker & $x / M$ & $-/+s$ & $x / M$ & $-/+s$ & $x / M$ & $-/+s$ & $x / M$ & $-/+s$ & $x / M$ & $-/+s$ & $x / M$ & $-/+s$ \\
\hline 1 & posture & 1.00 & & $3.00^{*}$ & & $3.00^{*}$ & & $3.00 *$ & & 3.00* & & $3.00 *$ & \\
\hline 2 & muscular tonus & 0.00 & & $2.00^{*}$ & & $2.00^{*}$ & & $2.00^{*}$ & & $2.00^{*}$ & & $2.00^{*}$ & \\
\hline 3 & rearing & 4.75 & 3.15 & $1.13^{*}$ & 1.89 & $\mathbf{0 . 1 3}^{*}$ & 1.35 & $1.25^{*}$ & 2.19 & 3.00 & 4.63 & $0.75^{*}$ & 1.49 \\
\hline 4 & hyperkinesis & 0.00 & & 0.00 & & $7.00^{*}$ & & 0.00 & & 0.00 & & $7.00^{*}$ & \\
\hline 5 & tremors & 0.00 & & 0.00 & & $5.00^{*}$ & & 0.00 & & 0.00 & & 0.00 & \\
\hline 6 & clonic movements & 0.00 & & $2.00^{*}$ & & $2.00^{*}$ & & 0.00 & & 0.00 & & $2.00 *$ & \\
\hline 7 & tonic movements & 0.00 & & 0.00 & & $5.00^{*}$ & & 0.00 & & 0.00 & & 0.00 & \\
\hline 8 & gait & 0.00 & & $7.00^{*}$ & & $7.00^{*}$ & & $7.00^{*}$ & & $5.00^{*}$ & & $3.00^{*}$ & \\
\hline 9 & ataxia & 0.00 & & $2.00^{*}$ & & $2.00^{*}$ & & $2.00^{*}$ & & 0.00 & & $2.00 *$ & \\
\hline 10 & gait score & 0.00 & & $2.00^{*}$ & & $2.00^{*}$ & & $2.00 *$ & & 0.00 & & $2.00^{*}$ & \\
\hline 11 & mobility score & 1.00 & & 4.00* & & $1.00^{*}$ & & 1.00 & & 1.00 & & 4.00* & \\
\hline 12 & activity & 4.00 & & $1.00^{*}$ & & $1.00^{*}$ & & $1.00^{*}$ & & 1.00 & & $1.00^{*}$ & \\
\hline 13 & RRF & 1.00 & & 1.00 & & $7.00^{*}$ & & 1.00 & & 1.00 & & $7.00^{*}$ & \\
\hline 14 & RRV & 1.00 & & 1.00 & & 4.00* & & 1.00 & & 1.00 & & 1.00 & \\
\hline 15 & landing foot splay (mm) & 83.75 & 7.94 & 43.19* & 38.33 & 30.88* & 33.71 & 40.69* & 28.14 & 69.13 & 35.52 & $42.25^{*}$ & 43.54 \\
\hline 16 & forelimb grip strenght $(\mathrm{kg})$ & 6.55 & 1.12 & $2.45^{*}$ & 2.32 & $1.73^{*}$ & 1.86 & $2.43^{*}$ & 1.75 & $3.95^{*}$ & 2.13 & $2.01^{*}$ & 2.24 \\
\hline 17 & hindlimb grip strenght $(\mathrm{kg})$ & 1.13 & 0.17 & $0.41^{*}$ & 0.37 & $0.25^{*}$ & 0.28 & $0.41^{*}$ & 0.27 & 0.71* & 0.38 & $0.43^{*}$ & 0.45 \\
\hline 18 & grip strength of all limbs $(\mathrm{kg})$ & 16.15 & 0.90 & $5.61^{*}$ & 5.56 & $3.28^{*}$ & 3.63 & $5.44^{*}$ & 3.80 & $10.35^{*}$ & 5.47 & $6.63^{*}$ & 8.06 \\
\hline 19 & vertical activity & 152.25 & 121.94 & $27.75^{*}$ & 47.28 & 35.88* & 92.72 & $48.88^{*}$ & 57.80 & $38.75^{*}$ & 53.26 & 63.88 & 73.76 \\
\hline 20 & horizontal activity & 18.25 & 30.33 & 0.13 & 0.35 & 0.63 & 1.77 & 3.13 & 5.94 & 5.00 & 9.27 & 9.00 & 20.09 \\
\hline 21 & total motor activity & 170.50 & 151.63 & $\mathbf{2 7 . 8 8}^{*}$ & 47.50 & 36.50 & 94.48 & 52.00 & 60.55 & 43.75* & 61.94 & 72.88 & 88.85 \\
\hline & & \multicolumn{2}{|c|}{$\mathrm{n}=8$} & \multicolumn{2}{|c|}{$\mathrm{n}=6$} & \multicolumn{2}{|c|}{$\mathrm{n}=5$} & \multicolumn{2}{|c|}{$\mathrm{n}=6$} & \multicolumn{2}{|c|}{$\mathrm{n}=7$} & \multicolumn{2}{|c|}{$\mathrm{n}=5$} \\
\hline
\end{tabular}


Tab. 2b: The values of cyclosarin-induced sensorimotor and excitability neurotoxic markers measured at $24 \mathrm{hr}$ following cyclosarin challenge according to the functional observational battery (scored values). Statistical significance: ${ }^{*} \mathrm{p}<0.05$ (comparison with the control values). Applied abbreviations - see Tab. 2a.

\begin{tabular}{|c|c|c|c|c|c|c|c|c|c|c|c|c|c|}
\hline \multicolumn{2}{|r|}{24 hours } & \multicolumn{2}{|c|}{ Controls } & \multicolumn{2}{|c|}{$\begin{array}{c}\text { Cyclosarin + A } \\
+ \text { K } 156\end{array}$} & \multicolumn{2}{|c|}{$\begin{array}{c}\text { Cyclosarin + A } \\
+ \text { K } 203\end{array}$} & \multicolumn{2}{|c|}{$\begin{array}{c}\text { Cyclosarin + A } \\
\text { + Obidoxime } \\
\end{array}$} & \multicolumn{2}{|c|}{$\begin{array}{c}\text { Cyclosarin + A } \\
+ \text { HI-6 } \\
\end{array}$} & \multicolumn{2}{|c|}{ Cyclosarin } \\
\hline No & Marker & $x / M$ & $-/+s$ & $x / M$ & $-/+s$ & $x / M$ & $-/+s$ & $x / M$ & $-/+s$ & $x / M$ & $-/+s$ & $x / M$ & $-/+s$ \\
\hline 1 & catch difficulty & 2.00 & & $1.00^{*}$ & & $1.00^{*}$ & & $3.00^{*}$ & & $1.00^{*}$ & & $1.00^{*}$ & \\
\hline 2 & ease of handling & 2.00 & & $1.00^{*}$ & & $1.00^{*}$ & & 2.00 & & $1.00^{*}$ & & $1.00^{*}$ & \\
\hline 3 & arousal (GSC) & 1.00 & & $4.00^{*}$ & & 4.00* & & $4.00^{*}$ & & $4.00^{*}$ & & $4.00^{*}$ & \\
\hline 4 & tension & 0.00 & & 0.00 & & 0.00 & & 0.00 & & 0.00 & & 0.00 & \\
\hline 5 & vocalisation & 0.00 & & 0.00 & & 0.00 & & 0.00 & & 0.00 & & 0.00 & \\
\hline 6 & stereotypy & 0.00 & & 0.00 & & 0.00 & & 0.00 & & 0.00 & & 0.00 & \\
\hline 7 & bizarre behavior & 0.00 & & 0.00 & & 0.00 & & 0.00 & & 0.00 & & 0.00 & \\
\hline 8 & approach response & 2.00 & & $1.00^{*}$ & & $1.00^{*}$ & & 2.00 & & $1.00^{*}$ & & $1.00^{*}$ & \\
\hline 9 & touch response & 2.00 & & 2.00 & & $1.00^{*}$ & & 2.00 & & 2.00 & & $1.00^{*}$ & \\
\hline 10 & click response & 2.00 & & 2.00 & & $1.00^{*}$ & & 2.00 & & 2.00 & & 2.00 & \\
\hline 11 & tail pinch response & 2.00 & & 2.00 & & $1.00^{*}$ & & $1.00^{*}$ & & 2.00 & & $1.00^{*}$ & \\
\hline & & \multicolumn{2}{|c|}{$\mathrm{n}=8$} & \multicolumn{2}{|c|}{$n=6$} & \multicolumn{2}{|c|}{$\mathrm{n}=5$} & \multicolumn{2}{|c|}{$\mathrm{n}=6$} & \multicolumn{2}{|c|}{$\mathrm{n}=7$} & \multicolumn{2}{|c|}{$n=5$} \\
\hline
\end{tabular}

Tab. 2c: The values of cyclosarin-induced autonomic neurotoxic markers measured at $24 \mathrm{hr}$ following cyclosarin challenge according to the functional observational battery (No 1-7, 10-11, 15 - scored values, No 8-9, 12-14 - values in absolute units). Statistical significance: * $\mathrm{p}<0.05$ (comparison with the control values). Applied abbreviations - see Tab. $2 \mathrm{a}$.

\begin{tabular}{|c|c|c|c|c|c|c|c|c|c|c|c|c|c|}
\hline & 24 hours & Con & rols & $\begin{array}{r}\text { Cyclosa } \\
+\mathrm{K}\end{array}$ & $\begin{array}{l}\text { rin }+A \\
156\end{array}$ & $\begin{array}{c}\text { Cyclosa } \\
+K\end{array}$ & $\begin{array}{l}\operatorname{arin}+A \\
203\end{array}$ & $\begin{array}{c}\text { Cyclosa } \\
\text { + Obid }\end{array}$ & $\begin{array}{l}\text { rin + A } \\
\text { loxime }\end{array}$ & $\begin{array}{r}\text { Cyclosa } \\
+\mathrm{H}\end{array}$ & $\begin{array}{l}\text { rin + A } \\
\text { I-6 }\end{array}$ & Cyclo & sarin \\
\hline No & Marker & $x / M$ & $-/+s$ & $x / M$ & $-/+s$ & $x / M$ & $-/+s$ & $x / M$ & $-/+s$ & $x / M$ & $-/+s$ & $x / M$ & $-/+s$ \\
\hline 1 & lacrimation & 0.00 & & 0.00 & & 4.00* & & 0.00 & & 0.00 & & 0.00 & \\
\hline 2 & palpebral closure & 1.00 & & 1.00 & & $3.00 *$ & & 1.00 & & 1.00 & & 1.00 & \\
\hline 3 & endo/exophtalmus & 0.00 & & 0.00 & & $-1.00^{*}$ & & 0.00 & & 0.00 & & $-1.00^{*}$ & \\
\hline 4 & fur abnormalities & 0.00 & & 0.00 & & 7.00* & & 0.00 & & 0.00 & & 0.00 & \\
\hline 5 & skin abnormalities & 0.00 & & 0.00 & & $3.00 *$ & & 0.00 & & 0.00 & & 0.00 & \\
\hline 6 & salivation & 0.00 & & 0.00 & & $2.00^{*}$ & & 0.00 & & 0.00 & & 0.00 & \\
\hline 7 & nose secretion & 0.00 & & 3.00* & & 3.00* & & $3.00^{*}$ & & $3.00^{*}$ & & $3.00^{*}$ & \\
\hline 8 & urination & 4.68 & 6.53 & 0.50 & 0.93 & 0.25 & 0.71 & 3.38 & 4.47 & 1.50 & 4.24 & 0.00 & 0.00 \\
\hline 9 & defecation & 0.00 & & 0.00 & & 0.00 & & 0.00 & & 0.00 & & 0.00 & \\
\hline 10 & pupil size & 0.00 & & $-2.00^{*}$ & & $-2.00^{*}$ & & $-1.00^{*}$ & & $-2.00^{*}$ & & $-2.00^{*}$ & \\
\hline 11 & pupil response & 1.00 & & $0.00^{*}$ & & 0.00* & & $0.50^{*}$ & & $0.00^{*}$ & & $0.00^{*}$ & \\
\hline 12 & food receiving $(\%)$ & 100.00 & 0.00 & $10.00^{*}$ & 9.26 & $5.25^{*}$ & 5.95 & $17.25^{*}$ & 11.00 & 24.13* & 10.19 & $18.75^{*}$ & 17.68 \\
\hline 13 & body weight (g) & 213.63 & 22.43 & 145.38 & 121.18 & 108.75 & 115.40 & $127.75^{*}$ & 79.80 & 192.00 & 82.18 & $109.63 *$ & 95.09 \\
\hline 14 & body termperature $\left({ }^{\circ} \mathrm{C}\right)$ & 37.59 & 0.25 & $35.96^{*}$ & 0.90 & 35.99* & 0.75 & $36.23^{*}$ & 0.32 & 37.29 & 0.37 & $36.85^{*}$ & 0.87 \\
\hline 15 & respiration & 0.00 & & 0.00 & & $-2.00^{*}$ & & 0.00 & & 0.00 & & 0.00 & \\
\hline & & $\mathrm{n}=$ & & $\mathrm{n}=$ & $=6$ & $\mathrm{n}=$ & $=5$ & $\mathrm{n}=$ & & $\mathrm{n}=$ & & & $=5$ \\
\hline
\end{tabular}

tion in the 24 observed parameters. Cyclosarin caused passive behavior of rats during handling and catching, miosis, endophtalmus, nose secretion and a decrease in muscular tone. The exploratory and rearing activity were significantly decreased, gait was seriously impaired, ataxia, hyperkinesis and clonic movements were observed. In addition, the rats poisoned with cyclosarin did not show any reaction during a reflex test consisting of recording each rat's response to the frontal approach of the blunt end of a pen, touch of the pen to the posterior flank and a pinch on the tail. Inability of the pupils to constrict in response to light was also demonstrated. Additionally, a significant decrease in the distance between hindpaws after a jump, forelimb and hindlimb grip strength, body temperature, food receiving and body weight was also observed at 24 hours following cyclosarin challenge. Spontaneous total motor activity was markedly reduced as well (Tab. 2a-c).

On the contrary to the newly developed oxime K203 which is completely ineffective to reduce cyclosarin-induced acute neurotoxicity, the newly developed oxime K156 as well as the currently available obidoxime and HI-6 in combination with atropine showed a slight potency to reduce cyclosarin-induced neurotoxic signs but were unable to eliminate many cyclosarin-induced signs of acute neuro- 
toxicity. Passive behavior of rats during catching and handling, miosis, nose secretion and a decrease in muscular tone were observed, the exploratory and rearing activity were significantly decreased, gait was impaired and ataxia was observed in cyclosarin-poisoned rats treated with K156, obidoxime or HI-6. An inability of the pupils to constrict in response to light, a significant decrease in the distance between hindpaws after a jump, forelimb and hindlimb grip strength, a decrease in body temperature and food receiving were observed at 24 hours following cyclosarin challenge and antidotal treatment with atropine in combination with K156, obidoxime or HI-6 as well (Tab. 2a-c).

\section{Discussion}

Nerve agents which contain cyclosarin appear to cause centrally mediated seizure activity that can rapidly progress to epileptic status and contribute to profound brain damage. Nerve agent-induced hyperstimulation of cholinergic muscarinic receptors in the brain can induce the first phase of centrally mediated seizures, whereas sustained seizures (status epilepticus) are probably associated with increased glutamatergic activity leading to excitotoxic damage, predominantly in the hippocampus, amygdala, piriform and entorhinal cortices (17). Thus, the exposure of experimental animals to nerve agents in convulsion-inducing doses may result in irreversible lesions in the CNS that can manifest as neurobehavioral effects in convulsion survivors (1). Therefore, the ability of antidotes to counteract the acute neurotoxic effects of nerve agents and prevent nerve agentpoisoned organisms from irreversible lesions in the CNS is very important for successful antidotal treatment of acute nerve agent poisonings.

Generally, the number of pyridinium rings, the position of an oxime group in the pyridinium ring and the number of methylene-group linking chains between two quaternary pyridinium rings in the molecule of reactivators play an important role in the reactivating and therapeutic efficacy of oximes $(10,20)$. Bisquaternary oximes have a higher affinity towards both intact and nerve agent-inhibited $\mathrm{AChE}$ and therefore a higher potency to reactivate nerve agent-inhibited AChE compared to monoquaternary oximes (10). The position of an oxime group in the pyridinium ring is also very important for the reactivating and therapeutic efficacy of oximes. It was demonstrated that $\mathrm{AChE}$ reactivators with the oxime group in position 2 are the best reactivators of cyclosarin-inhibited $\operatorname{AChE}(8,12)$. On the other hand, the number of aldoxime groups is not so important. The oxime HI-6 has only one oxime group but it is significantly more efficacious to reactivate cyclosarin-inhibited AChE than bispyridinium oximes with two aldoxime groups, such as obidoxime, methoxime or trimedoxime (6, 9 ). The chain linking the two quaternary nitrogens in bispyridinium oximes exerts a great influence on the reactivating and therapeutic efficacy, although this part of the oxime reactivator molecule does not play any role in the de- phosphorylation process. It is a major factor in influencing oxime access and reactivation rates. Tri- or tetra-carbon chains seem to be the most suitable for the sufficient potency of oximes to reactivate cyclosarin-inhibited AChE and to counteract cyclosarin-induced acute toxicity $(10,24)$.

Our results demonstrate that the neuroprotective efficacy of newly developed oxime K156 is slightly higher compared to K203 but its potency to reduce cyclosarin-induced neurotoxic signs and symptoms is relatively low. In addition, some cyclosarin-induced neurotoxic signs and symptoms were even more intensive in comparison with non-treated cyclosarin-poisoned rats in the case of treatment of rats with the oxime K203. This fact could be explained by the adverse effect of phosphorylated oxime originated during dephosphorylation of the complex enzyme-inhibitor. Generally, the phosphorylated oxime is hydrolyzed very quickly but in certain situations the rapidity of hydrolysis of phosphorylated oximes is not high enough to protect reactivated AChE from reinhibition (24). The low neuroprotective efficacy of both the newly developed oximes is probably connected with their chemical structure, which is advantageous for their potency to counteract tabun-induced acute toxicity but is disadvantageous for their ability to counteract cyclosarin-induced acute toxicity. This is due to the fact that the oxime group of both newly synthesized oximes is situated in position 4. The oxime HI-6 having an oxime group in position 2 generally showed markedly higher reactivating and therapeutic efficacy against cyclosarin than other commonly used oximes $(6,8,9)$, although the differences between the neuroprotective efficacy of the oxime HI-6 and other oximes studied are not as high in this study.

In the case of human poisonings with organophosphorous compounds, an antidotal treatment usually consists of an anticholinergic drug to block acetlycholine-induced overstimulation of cholinergic muscarinic receptors (preferably atropine), an oxime to reactivate AChE inhibited by organophosphorous compound and an anticonvulsive drug to counteract centrally mediated seizures and tonic-clonic convulsions (15). Among oximes, pralidoxime and obidoxime are mostly used to reactivate nerve agents or organophosphorous insecticide-inhibited AChE but the effectiveness of both commonly used oximes is not so high, especially against highly toxic organophosphorous compounds called nerve agents. Therefore, a new generation of oximes, called $\mathrm{H}$ oximes, have been developed to increase the reactivating, therapeutical and neuroprotective effects of antidotal treatment of human poisonings. Among $\mathrm{H}$ oximes, the oxime HI-6 was considered to be the most promising and significantly more effective than obidoxime and pralidoxime against nerve agents with the exception of tabun. Nevertheless, HI-6 is as yet only licensed for human use in three countries (Sweden, Canada and Czech Republic), (14, 16).

In conclusion, due to their low potency to reduce cyclosarin-induced neurotoxic signs and symptoms, none of the newly developed oximes studied is a suitable AChE reacti- 
vator for replacing the commonly used oximes for the antidotal treatment after exposure to cyclosarin. On the other hand, the oxime HI- 6 can be still considered to be the best oxime for the antidotal treatment of acute poisonings with cyclosarin and it should be licensed for human use to replace pralidoxime and obidoxime (23).

\section{Acknowledgements}

The authors would like to thank to Mrs. E. Reslova and Mrs. J. Uhlirova for their skilful technical assistance. The study was supported by a grant from the Ministry of Defense, FVZMO0000501.

\section{References}

1. Bajgar J. Organophosphate/nerve agent poisoning: mechanism of action, diagnosis, prophylaxis, and treatment. Adv Clin Chem 2004:38:151-216.

2. Cassel G, Karlsson L, Waara L, Wee Ang K, Goransson-Nyberg A. Pharmacokinetics and effects of HI-6 in blood and brain of soman-intoxicated rats: a microdialysis study. Eur J Pharmacol 1997;332:43-52.

3. Frantík E, Hornychová M. Clustering of neurobehavioral measures of toxicity. Homeostasis 1995;36:19-25.

4. Hornychová M, Frantík E, Kubát J, Formánek J. Neurotoxicity profile of supermethrin, a new pyrethroid insecticide. Cent Eur J Publ Health 1995;3:210- 8

5. Kassa J. Review of oximes in the antidotal treatment of poisoning by organophosphorus nerve agents. J Toxicol Clin Toxicol 2002;40:803-16.

6. Kassa J, Cabal J. A comparison of the efficacy of acetylcholinesterase reactivators against cyclohexyl methylphosphonofluoridate (GF agent) by in vitro and in vivo methods. Pharmacol Toxicol 1999;84:41-5.

7. Kassa J, Karasová J, Musílek K, Kuča K. An evaluation of therapeutic and reactivating effects of newly developed oximes (K156, K203) and commonly used oximes (obidoxime, trimedoxime, HI-6) in tabun-poisoned rats and mice. Toxicology 2008;243:311-6.

8. Kuča K, Cabal J, Jun D, Bajgar J, Hrabinová M. Potency of new structurally different oximes to reactivate cyclosarin-inhibited human brain acetylcholinesterase. J Enz Inhib Med Chem 2006;21:663-6.
9. Kuča K, Cabal J, Jun D, et al. In vitro reactivation potency of some acetylcholinesterase reactivators against sarin- and cyclosarin-induced inhibitions. J App Toxicol 2005;25:296-300.

10. Kuča K, Jun D, Musílek K. Structural requirements of acetylcholinesterase reactivators. Mini-Rev Med Chem 2006;6:109-20.

11. Kuča K, Musilek K, Paar M, et al. Targeted synthesis of 1-(4-hydroxyiminomethylpyridinium)-3-pyridinium propane dibromide - a new nerve agent reactivator. Molecules 2007;12:1964-72.

12. Kuča J, Patočka J. Reactivation of cyclosarin-inhibited rat brain acetylcholinesterase by pyridinium-oximes. J Enz Inhib Med Chem 2004;19:39-43.

13. Lotti M. Organophosphorus compounds. In: Spencer PS, Schaumburg HH (eds) Experimental and Clinical Neurotoxicology. New York: Oxford University Press 2000:898-925.

14. Lundy PM, Raved L, Amitai G. Development of the bisquaternary oxime HI-6 toward clinical use in the treatment of organophosphate nerve agent poisoning. Toxicol Rev 2006;25:231-43.

15. Marrs TC. Organophosphate poisoning. Pharmacol Ther 1993;58:51-66.

16. Marrs TC, Rice P, Vale JA. The role of oximes in the treatment of nerve agent poisoning in civilian casualties. Toxicol Rev 2006;25:297-323.

17. McDonough JH Jr, Shih T-M Neuropharmacological modulation of nerve agent-induced seizure and neuropathology. Neurosci Biobehav Rev 1997;21: 559-79.

18. Moser VC, Tilson H, McPhail RC, et al. The IPCS collaborative study on neurobehavioral screening methods: II. Protocol design and testing procedures. Neurotoxicology 1997;18:929-38.

19. Musilek K, Holas O, Kuča K, et al. Synthesis of monooxime-monocarbamoy bispyridinium compounds bearing (E)-but-2-ene linker and evaluation of their reactivation activity against tabun- and paraoxon-inhibited acetylcholinesterase J. Enz. Inhib. Med. Chem. 2008; 23:70-6.

20. Musilek K, Kuča K, Jun D, Doležal M. Progress in synthesis of new acetylcholinesterase reactivators during the period 1990-2004. Curr Org Chem 2007; $11 \cdot 229-38$

21. Sakurada K, Matsubara K, Shimizu K, et al. Pralidoxime iodide (2-PAM) penetrates across the blood-brain barrier. Neurochem Res 2003;28:1401-7.

22. Taylor P. Anticholinesterase agents. In: Hardman JG, Limbird LE (eds). The Pharmacological Basis of Therapeutics. New York: McGraw Hill 1996:161-76.

23. Wiener SW, Hoffman RS. Nerve agents: a comprehensive review. J Intensive Care Med 2004;19:22-37.

24. Worek F, Eyer P, Szinicz L. Inhibition, reactivation and aging kinetics of cyclohexylphosphonofluoridate-inhibited human cholinesterases. Arch Toxicol 1998; 72:580-7.

Submitted August 2008 . Accepted December 2008.

\section{Corresponding author:}

Prof. Jiří Kassa, M.D., CSc., Trebešská 1575, Faculty of Military Health Sciences, 50001 Hradec Králové, Czech Republic; e-mail:kassa@pmfhk.cz 